\title{
Preoperative controlling nutritional status (CONUT) score as a predictor of long-term outcome after curative resection followed by adjuvant chemotherapy in stage II-III gastric Cancer
}

Xuechao Liu ${ }^{1,2+}$, Deyao Zhang ${ }^{1,2+}$, Enzi Lin ${ }^{3+}$, Yongming Chen ${ }^{1,2}$, Wei Li ${ }^{1,2}$, Yingbo Chen ${ }^{1,2}$, Xiaowei Sun ${ }^{1,2^{*}}$ and Zhiwei Zhou ${ }^{1,2^{*}}$ (D)

\begin{abstract}
Background: The prognostic value of preoperative controlling nutritional status (CONUT) has been reported in many malignancies. In present study, we aimed to clarify the prognostic impact of CONUT in gastric cancer (GC) receiving curative resection and adjuvant chemotherapy.

Methods: We retrospectively reviewed 697 consecutive patients undergoing curative surgery followed by adjuvant chemotherapy for Stage II-III GC between November 2000 and September 2012. Patients were classified into high $(\geq 3)$ and low ( $\leq 2)$ CONUT groups according to the receiver operating characteristic (ROC) analysis.

Results: Of the included patients, 217 (31.1\%) belonged to the high CONUT group. The high CONUT group had a significantly lower 5 -year cancer-specific survival (CSS) rate than the low CONUT group (39.3 vs. 55.5\%, $P<0.001$ ). High CONUT score was significantly associated with larger tumor size, more lymph node metastasis, and poorer nutritional status, including lower body mass index (BMI), higher prognostic nutritional index (PNI) and the presence of preoperative anemia (all $P<0.05$ ). Multivariate analysis revealed that CONUT score was an independent prognostic factor (HR: 1.553; 95\% Cl: 1.080-2.232; $P=0.017$ ). Of note, in the low PNI group, CONUT score still effectively stratified CSS $(P=0.016)$. Furthermore, the prognostic significance of CONUT score was also maintained when stratified by TNM stage (all $P<0.05$ ).
\end{abstract}

Conclusions: CONUT score is considered a useful nutritional marker for predicting prognosis in stage II-III GC patients undergoing curative resection and adjuvant chemotherapy, and may help to facilitate the planning of preoperative nutritional interventions.

Keywords: CONUT score, PNI, Adjuvant chemotherapy, Gastric cancer, Prognosis

\footnotetext{
* Correspondence: sunxw@sysucc.org.cn; zhouzhw@sysucc.org.cn

${ }^{\dagger}$ Xuechao Liu, Deyao Zhang and Enzi Lin contributed equally to this work.

${ }^{1}$ State Key Laboratory of Oncology in South China; Collaborative Innovation

Center for Cancer Medicine, Sun Yat-sen University Cancer Center,

Guangzhou 510060, China

Full list of author information is available at the end of the article
}

(c) The Author(s). 2018 Open Access This article is distributed under the terms of the Creative Commons Attribution 4.0 International License (http://creativecommons.org/licenses/by/4.0/), which permits unrestricted use, distribution, and reproduction in any medium, provided you give appropriate credit to the original author(s) and the source, provide a link to the Creative Commons license, and indicate if changes were made. The Creative Commons Public Domain Dedication waiver (http://creativecommons.org/publicdomain/zero/1.0/) applies to the data made available in this article, unless otherwise stated. 


\section{Background}

Gastric cancer (GC) is the third most common cause of cancer death and a major public health problem worldwide. In China, despite the decreasing incidence trend of GC, population growth and ageing still lead to a large and rising number of new cases in recent years $[1,2]$. To better achieve the clinical outcome, surgical technique, chemotherapies and targeted therapy have improved [3]. Recently, there is increasing interest for clinicians to identify prognostic factors for tailored treatment.

One such factor that has arisen substantial attention is the nutritional and immunological status, which is reported to be associated with the clinical outcomes in various malignancies [4-6]. Several preoperative scoring systems are developed to assess nutritional risk, postoperative complications and long-term outcomes, such as the prognostic nutritional index (PNI), subjective global assessment, and Nutritional Risk Index [7-9]. The controlling nutritional status (CONUT) score, another screening tool for nutritional status, is calculated from the serum albumin concentration, total cholesterol level and total peripheral lymphocyte count, which are representative markers of protein reserves, calorie deficiency, and impaired immune defenses, respectively [10]. Serum albumin concentration is not only a major indicator of nutritional status but also an important determinant of the immune response. Hypoalbuminemia has been reported to be associated with poor outcome in various malignancies, including GC $[11,12]$. Total cholesterol level also has been revealed to correlate with tumour progression and prognosis in many types of cancers [13]. In addition, lymphocytes play a key role in cell-mediated immunity and are thought to initiate a cytotoxic immune response by inducing cell apoptosis, suppressing tumor cell proliferation, invasion, and migration [14]. The combination of the three components into CONUT may better reflect the balance of nutritional status and enhance the ability to accurately predict general condition.

Recently, CONUT score has been demonstrated as a predictive or prognostic marker in many types of cancers [15-17]. Of note, a study from Japan showed that CONUT was useful for predicting long-term outcome in pStage I-II, but not in pStage III GC patients [18]. Due to the regional differences as well as different multidisciplinary treatment mode, the impact of CONUT score on prognosis in GC patients undergoing curative resection and adjuvant chemotherapy remains unclear.

In this study, we performed a sufficiently large, representative and consecutive sample to evaluate the prognostic value of the preoperative CONUT score, along with several common nutritional markers including PNI, body mass index (BMI), performance status and preoperative anemia.

\section{Material and methods \\ Patients}

We retrospectively reviewed the medical records of 697 consecutive patients undergoing open D2 radical gastrectomy with R0 resection at Sun Yat-sen University Cancer Center, from November 2000 to September 2012. All patients had histologically confirmed stage II-III gastric adenocarcinoma, as defined by the seventh edition of the American Joint Committee on Cancer (AJCC) tumornodes-metastasis (TNM) classification. By multidisciplinary discussion, eligible patients had no marked comorbidities that would preclude the use of adjuvant chemotherapy. After surgery, all patient routinely received 5-fluorouracilbased (5-FU) adjuvant chemotherapy for more than four cycles $[19,20]$. In principle, patients were treated until disease progression or unacceptable side effects occurred. Adjuvant chemotherapy was administered by the intravenous route or orally, as appropriate for the specific regimen.

Exclusion criteria were as follows: 1) incomplete clinical and laboratory data; 2) neoadjuvant chemotherapy or radiotherapy; 3 ) other adjuvant chemotherapy or radiotherapy; 4) preoperative parenteral nutrition before the blood sample was taken. Ultimately, 697 patients were enrolled.

Clinical and laboratory data were retrospectively obtained from an electronic database and the medical records of each patient. The Sun Yat-sen University Cancer Center research ethics committee approved this study that was conducted in accordance with the standards of the Declaration of Helsinki. Informed consent was deemed unnecessary by the Ethical Committee, and all information were anonymous.

\section{Follow-up strategy}

All patients were routinely followed up every 3 months for the first 2 years, every 6 months for the next 3 years, and annually thereafter. Postoperative follow-up procedures included medical checkups, laboratory testing, gastroscope examination, and chest/abdominal computed tomography scan. All patients were monitored either until July 2015 or their death. Median follow-up time was 36 months (range, 3-162 months). Cancer-specific survival (CSS) was calculated from the date of operation until death of $\mathrm{GC}$ or last follow-up.

\section{CONUT score and other markers}

Preoperative blood samples were collected and assayed within 2 weeks before surgery. Preoperative CONUT scores were summarized in Table 1 [17]. We set 3 as the optimal cutoff value for CONUT score by receiver operating characteristics (ROC) curves analysis (Additional file 1: Figure S1). BMI, PNI and performance status were calculated and classified based on previous studies [18, 21]. Patients with a combined albumin $(\mathrm{g} / \mathrm{L}) \times$ total lymphocyte 
Table 1 Assessment of the nutritional status according to the CONUT score

\begin{tabular}{|c|c|c|c|c|}
\hline & None & Light & Moderate & Severe \\
\hline Serum albumin (g/dL) & $\geq 3.50$ & $3.00-3.49$ & $2.50-2.99$ & $<2.50$ \\
\hline Score & 0 & 2 & 4 & 6 \\
\hline Total lymphocyte count $\left(/ \mathrm{mm}^{3}\right)$ & $\geq 1600$ & 1200-1599 & 800-1199 & $<800$ \\
\hline Score & 0 & 1 & 2 & 3 \\
\hline Total cholesterol (mg/dL) & $>180$ & $140-179$ & $100-139$ & $<100$ \\
\hline Score & 0 & 1 & 2 & 3 \\
\hline CONUT score (total) & $0-1$ & $2-4$ & $5-8$ & $9-12$ \\
\hline \multirow[t]{2}{*}{ Classification (total score) } & \multicolumn{4}{|c|}{$\leq 2$ Low CONUT group } \\
\hline & \multicolumn{4}{|c|}{$\geq 3$ High CONUT group } \\
\hline
\end{tabular}

Abbreviations: CONUT controlling nutritional status

count $\times 10^{9} / \mathrm{L} \geq 45$ were allocated a PNI score of 0 . Patients in whom this total score was $<45$ were allocated a score of 1 , where a PNI of 1 is indicative of severe nutritional impairment and PNI of 0 is normal [21]. According to the manufacturer's instructions, the cutoff values for elevated concentrations of serum carcinoembryonic antigen (CEA), carbohydrate antigen (CA) 19-9 and $\mathrm{CA} 72-4$ were $5 \mathrm{ng} / \mathrm{mL}, 27 \mathrm{U} / \mathrm{mL}$, and $5 \mathrm{U} / \mathrm{mL}$, respectively.

\section{Statistical methods}

Our research adhered to the Strengthening the Reporting of Observational Studies in Epidemiology (STROBE) statement (Additional file 2: Table S1). The CSS rate was estimated by the Kaplan-Meier method with the log-rank test. Differences between groups were examined using the Chi-square test for categorical variables. The optimal cutoff value was determined by the maximum of Youden index (sensitivity+ specificity-1) based on ROC curve analyses. The variables in which $p$ value was less than 0.05 in the univariate analysis were entered into a final multivariate Cox proportional hazards model to identify independent prognostic factors. A two-sided $P$ value $<0.05$ was considered to be statistically significant. All of the statistical analyses were performed with SPSS version 19.0 (SPSS, Chicago, IL, USA). All data in our study have been recorded at Sun Yat-sen University Cancer Center for future reference (number RDDA2018000485).

\section{Results}

Of the 697 enrolled patients, 194 (27.8\%) were classified as stage II and $503(72.2 \%)$ as stage III. The patient cohort included 457 (65.6\%) male patients, with a median age of 57 years (range, 21-86 years) and the mean age was 66.0 years (range $41-89$ years). According to the nutritional status in CONUT score, the patients were divided into four groups: none (261 patients, 37.4\%), light (396 patients, $56.8 \%$ ), moderate ( 39 patients, $5.6 \%)$, and severe (1 patients,
0.1\%) (Table 1; Fig. 1). Finally, 480 (68.9\%) patients were classified into the low CONUT group and 217 (31.1\%) patients were classified into the high CONUT group based on a cut-off CONUT value of 3. The Kaplan-Meier curve comparing the CSS of the patients according to the CONUT score is shown in Fig. 2.The high CONUT group had a significantly lower 5-year CSS rate than the low CONUT group (39.3 vs. $55.5 \%, P<0.001)$.

The correlation between the CONUT and the clinicopathological factors is shown in Table 2. High CONUT group was significantly associated with larger tumor size $(P=0.002)$, more lymph node metastasis $(P=0.010)$, lower BMI $(P=0.009)$, higher PNI $(P<0.001)$ and the presence of preoperative anemia $(P<0.001)$.

The results of univariate analyses showed that age, tumor size, tumor location, lymphatic vessel infiltration

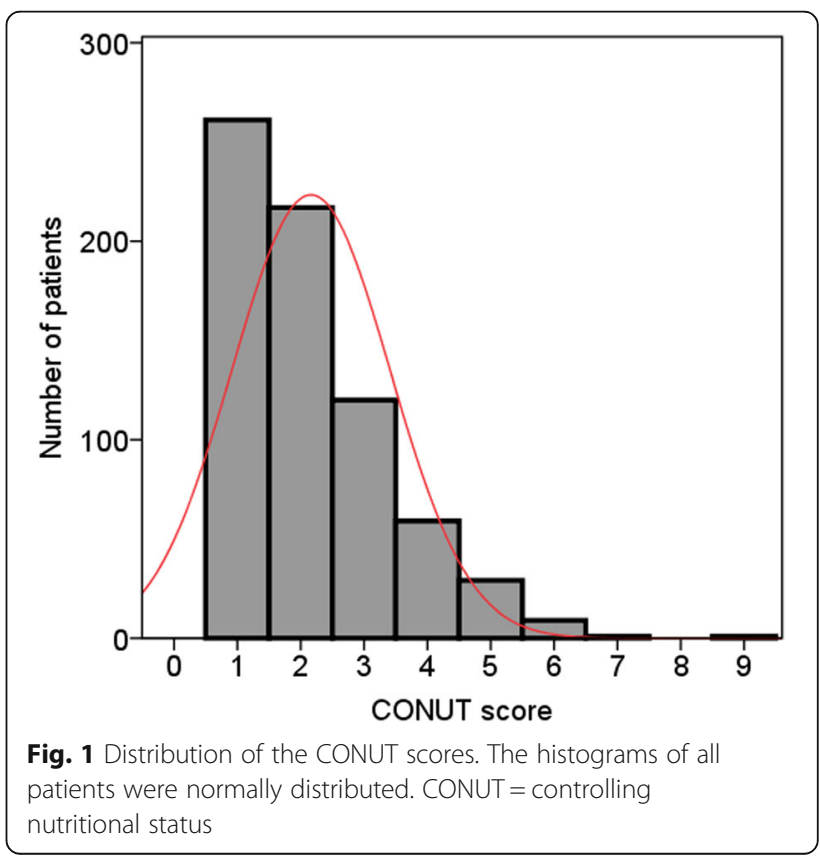




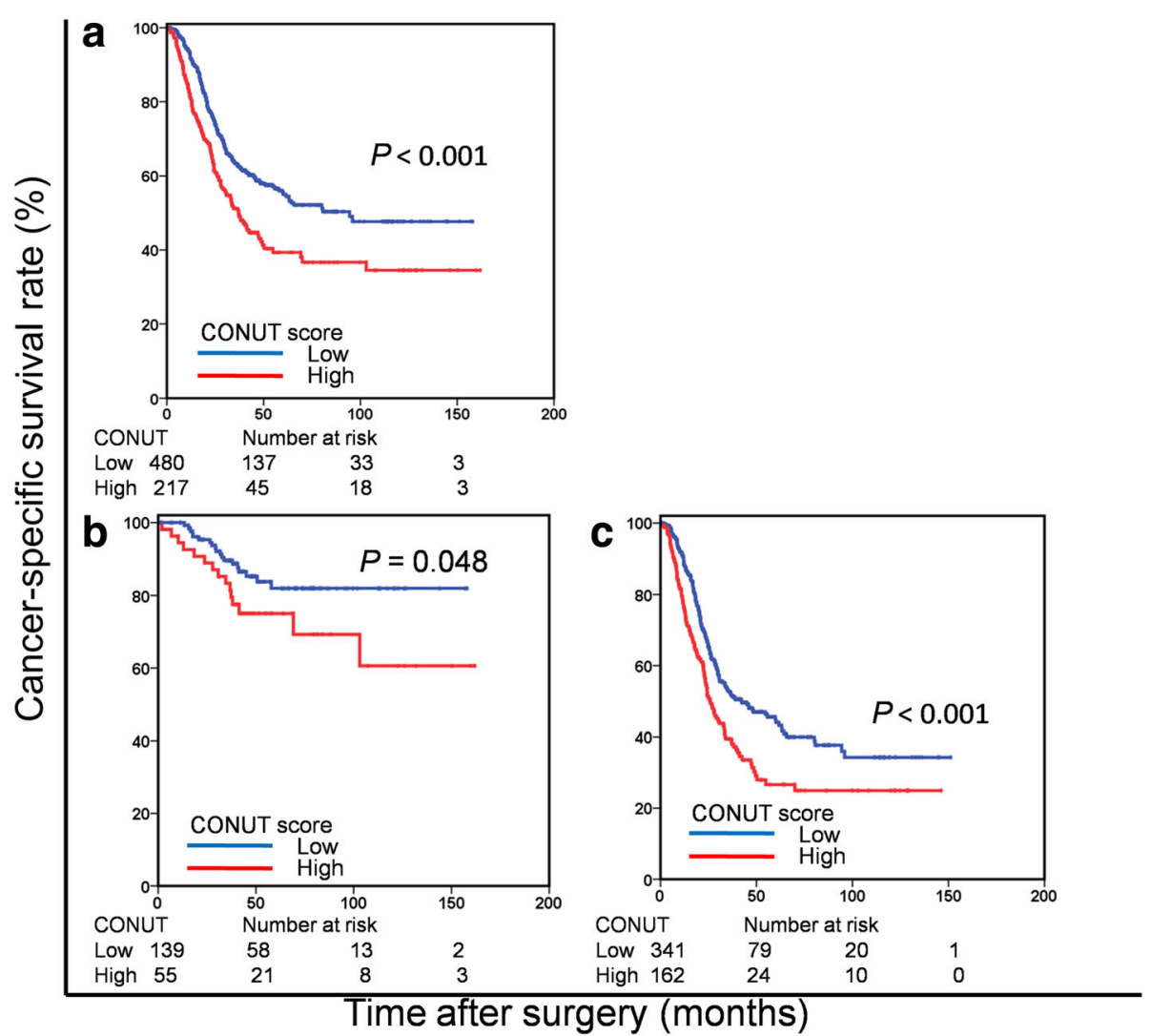

Fig. 2 Cancer-specific survival based on the CONUT score in patients with stage II-III (a), stage II (b), and stage III (c) gastric cancer, respectively. CONUT = controlling nutritional status

(LVI), pT stage, pN stage, TNM stage, operation type, PNI, CONUT, CEA, CA19-9, and CA72-4 were associated with CSS (All $P<0.05$; Table 3). Considering that $\mathrm{pT} / \mathrm{pN}$ stages were significantly associated with TNM stage, we didn't include them in the final multivariable analysis. When a multivariate analysis was performed, CONUT score were independent predictors of CSS (HR: 1.553; 95\% CI: $1.080-2.232 ; P=0.017$ ), along with tumor location, LVI, TNM stage and CA19-9.

When stratified by TNM stage, the prognostic significance of CONUT score was also maintained in patients with stage II $(P=0.048)$ and stage III $(P<0.001)$ GC. Furthermore, we found that 137 patients $(22.2 \%)$ belonged to the low PNI group and the high CONUT group. Of note, in the low PNI group, CONUT score still effectively stratified CSS $(P=0.016$; Fig. 3$)$.

\section{Discussion}

Cancer-associated malnutrition is a common but usually unemphasized problem, especially in gastrointestinal malignancies [22]. Increasing evidence has been gathered by clinicians suggesting that malnutrition is closely associated with various clinical consequences, including poor life quality, decreased response to chemotherapy, and the incidence of severe toxicity during adjuvant therapy. Subsequently, severe adverse events often result in decreased oral food intake, treatment schedule modification or interruptions, and greater impairment of life quality, which lead to further malnutrition [23]. In fact, in recent years, it has been well acceptable that malnutrition is associated with poor clinical outcomes [24]. Therefore, clinicians also continue to seek reliable biomarkers for identifying cancer-associated malnutrition and improving the clinical management.

Recently, the presence of immune-nutritional status, as indicated by the CONUT score, has been reported to independently predict prognosis in many malignancies [25]. In present study, we determined the prognostic value of the preoperative CONUT score, along with several common nutritional markers including PNI, BMI, performance status and preoperative anemia, in stage II-III GC patients receiving adjuvant chemotherapy. We found the CONUT score was a independent predictor of outcome in these patients, which appeared to be a superior prognostic marker compared with the other nutritional markers we tested. 
Table 2 The clinicopathological characteristics stratified by the cONUT score

\begin{tabular}{|c|c|c|c|}
\hline & $\begin{array}{l}\text { Low CONUT } \\
\text { group } \\
(n=480)\end{array}$ & $\begin{array}{l}\text { High CONUT } \\
\text { group } \\
(n=217)\end{array}$ & $P$ value \\
\hline Age (years) & & & 0.070 \\
\hline$<60$ & 296 & 118 & \\
\hline$\geq 60$ & 184 & 99 & \\
\hline Sex & & & 0.247 \\
\hline Female & 172 & 68 & \\
\hline Male & 308 & 149 & \\
\hline Tumor size (cm) & & & 0.002 \\
\hline$<5$ & 261 & 91 & \\
\hline$\geq 5$ & 219 & 126 & \\
\hline Tumor location & & & 0.062 \\
\hline Lower third & 183 & 99 & \\
\hline Upper/Middle third & 297 & 118 & \\
\hline Histological grade & & & 0.053 \\
\hline Well differentiated & 56 & 37 & \\
\hline $\begin{array}{l}\text { Poorly } \\
\text { differentiated }\end{array}$ & 424 & 180 & \\
\hline Lauren histotype & & & 0.403 \\
\hline Intestinal & 103 & 59 & \\
\hline Diffuse / Mixed & 221 & 107 & \\
\hline LVI & & & 0.096 \\
\hline Absent & 362 & 106 & \\
\hline Present & 27 & 14 & \\
\hline pT stage & & & 0.134 \\
\hline $\mathrm{pT} 1 / 2$ & 45 & 13 & \\
\hline $\mathrm{pT3} / 4$ & 435 & 204 & \\
\hline pN stage & & & 0.010 \\
\hline $\mathrm{pNO} / 1$ & 190 & 64 & \\
\hline $\mathrm{pN} 2 / 3$ & 290 & 153 & \\
\hline $\begin{array}{l}\text { Dissected lymph } \\
\text { nodes }\end{array}$ & & & 0.090 \\
\hline$\leq 29$ & 355 & 147 & \\
\hline$>29$ & 125 & 70 & \\
\hline TNM stage & & & 0.324 \\
\hline$\|$ & 139 & 55 & \\
\hline III & 341 & 162 & \\
\hline Operation type & & & 0.109 \\
\hline Subtotal & 332 & 163 & \\
\hline Total/extended & 148 & 54 & \\
\hline Complications & & & 0.161 \\
\hline No & 375 & 159 & \\
\hline Yes & 105 & 58 & \\
\hline
\end{tabular}

Table 2 The clinicopathological characteristics stratified by the CONUT score (Continued)

\begin{tabular}{|c|c|c|c|}
\hline & $\begin{array}{l}\text { Low CONUT } \\
\text { group } \\
(n=480)\end{array}$ & $\begin{array}{l}\text { High CONUT } \\
\text { group } \\
(n=217)\end{array}$ & $P$ value \\
\hline Performance status & & & 0.527 \\
\hline 0 & 119 & 49 & \\
\hline $1 / 2$ & 361 & 168 & \\
\hline BMI (Kg/m2) & & & 0.009 \\
\hline$<18.5$ & 294 & 110 & \\
\hline $18.5 \leq$ & 186 & 107 & \\
\hline $\mathrm{PNI}$ & & & $<0.001$ \\
\hline$\geq 45$ & 480 & 137 & \\
\hline$<45$ & 0 & 80 & \\
\hline Anemia & & & $<0.001$ \\
\hline No & 378 & 110 & \\
\hline Yes & 102 & 107 & \\
\hline CEA & & & 0.164 \\
\hline Normal & 381 & 162 & \\
\hline Elevated & 99 & 55 & \\
\hline CA19-9 & & & 0.633 \\
\hline Normal & 375 & 166 & \\
\hline Elevated & 105 & 51 & \\
\hline CA72-4 & & & 0.738 \\
\hline Normal & 364 & 161 & \\
\hline Elevated & 116 & 56 & \\
\hline
\end{tabular}

Abbreviations: CONUT controlling nutritional status, LVI lymphatic vessel infiltration, TNM tumor-node-metastasis staging, $B M I$ body mass index, $P N I$ prognostic nutritional index, CEA carcinoembryonic antigen, $C A$ carbohydrate antigen

Recently, a Japanese study reported, in a series of 416 $\mathrm{GC}$, that CONUT score was retained as an independent prognostic marker in pStage I-II, but not in pStage III GC patients. It should be noted that, in this study, most of patients were early GC and only $14.4 \%$ patients were classified as pStage III [18]. As we all know, GC in Japan is often detected at an early stage and has less aggressive clinicopathological features and better prognosis than those from China [26]. Furthermore, under the Japanese social security system, there are fewer problems of cancer-associated malnutrition and unaffordable medical care in Japan. Therefore, our study is needed to further validate the prognostic value of CONUT score in China.

In fact, our conclusions are supported by other studies. Iseki $\mathrm{Y}$ et al. reported that the CONUT score was a strong independent predictor of outcomes among colorectal cancer patients and it more accurately predicted prognosis in those patients than the PNI [27]. The PNI, as a promising 
Table 3 Univariate and multivariate analyses of prognostic factors associated with cancer-specific survival

\begin{tabular}{|c|c|c|}
\hline & Univariate analysis & Multivariate analysis \\
\hline & HR (95\% Cl) P-value & HR (95\% Cl) P-value \\
\hline Age (years) & 0.024 & 0.421 \\
\hline$<60$ & 1.00 & 1.00 \\
\hline$\geq 60$ & $1.292(1.035,1.613)$ & $1.130(0.839,1.522)$ \\
\hline Sex & 0.252 & \\
\hline Female & 1.00 & \\
\hline Male & $0.875(0.695,1.100)$ & \\
\hline Tumor size (cm) & $<0.001$ & 0.997 \\
\hline$<5$ & 1.00 & 1.00 \\
\hline$\geq 5$ & $1.505(1.204,1.880)$ & $0.999(0.737,1.356)$ \\
\hline Tumor location & $<0.001$ & 0.027 \\
\hline Lower third & 1.00 & 1.00 \\
\hline Upper/Middle third & $1.524(1.209,1.922)$ & $1.453(1.044,2.022)$ \\
\hline Histological grade & 0.340 & \\
\hline Well differentiated & 1.00 & \\
\hline Poorly differentiated & $1.179(0.840,1.655)$ & \\
\hline Lauren histotype & 0.235 & \\
\hline Intestinal & 1.00 & \\
\hline Diffuse / Mixed & $0.859(0.669,1.104)$ & \\
\hline LVI & $<0.001$ & 0.041 \\
\hline Absent & 1.00 & 1.00 \\
\hline Present & $1.602(1.299,1.977)$ & $1.294(1.011,1.657)$ \\
\hline pT stage & 0.001 & \\
\hline $\mathrm{p} T 1 / 2$ & 1.00 & \\
\hline $\mathrm{pT} 3 / 4$ & $2.960(1.573,5.571)$ & \\
\hline pN stage & $<0.001$ & \\
\hline $\mathrm{pNO} / 1$ & 1.00 & \\
\hline $\mathrm{pN} 2 / 3$ & $3.743(2.734,5.124)$ & \\
\hline Dissected lymph nodes & 0.118 & \\
\hline$\leq 29$ & 1.00 & \\
\hline$>29$ & $1.107(0.974,1.258)$ & \\
\hline TNM stage & $<0.001$ & $<0.001$ \\
\hline$\|$ & 1.00 & 1.00 \\
\hline III & $4.597(3.218,6.567)$ & $4.625(2.883,7.421)$ \\
\hline Operation type & 0.015 & 0.669 \\
\hline Subtotal & 1.00 & 1.00 \\
\hline Total/extended & $1.340(1.058,1.697)$ & $1.083(0.752,1.558)$ \\
\hline Complications & 0.755 & \\
\hline No & 1.00 & \\
\hline Yes & $0.953(0.701,1.293)$ & \\
\hline Performance status & 0.548 & \\
\hline 0 & 1.00 & \\
\hline $1 / 2$ & $0.924(0.716,1.194)$ & \\
\hline
\end{tabular}

Table 3 Univariate and multivariate analyses of prognostic factors associated with cancer-specific survival (Continued)

\begin{tabular}{|c|c|c|}
\hline & Univariate analysis & Multivariate analysis \\
\hline BMI $(\mathrm{Kg} / \mathrm{m} 2)$ & 0.159 & \\
\hline$<18.5$ & 1.00 & \\
\hline $18.5 \leq$ & $1.173(0.940,1.464)$ & \\
\hline $\mathrm{PNI}$ & $<0.001$ & 0.085 \\
\hline$\geq 45$ & 1.00 & 1.00 \\
\hline$<45$ & $1.777(1.313,2.404)$ & $1.505(0.945,2.396)$ \\
\hline Anemia & 0.231 & \\
\hline No & 1.00 & \\
\hline Yes & $1.155(0.912,1.463)$ & \\
\hline CONUT & $<0.001$ & 0.017 \\
\hline$\leq 2$ & 1.00 & 1.00 \\
\hline$\geq 3$ & $1.576(1.255,1.978)$ & $1.553(1.080,2.232)$ \\
\hline CEA & 0.009 & 0.269 \\
\hline Normal & 1.00 & 1.00 \\
\hline Elevated & $1.407(1.087,1.822)$ & $1.222(0.857,1.742)$ \\
\hline CA19-9 & 0.002 & 0.033 \\
\hline Normal & 1.00 & 1.00 \\
\hline Elevated & $1.555(1.183,2.042)$ & $1.427(1.029,1.979)$ \\
\hline CA72-4 & 0.012 & 0.476 \\
\hline Normal & 1.00 & 1.00 \\
\hline Elevated & $1.447(1.086,1.927)$ & $1.125(0.814,1.555)$ \\
\hline
\end{tabular}

Abbreviations: $L V I$ lymphatic vessel infiltration, TNM tumor-node-metastasis staging, $B M I$ body mass index, $P N I$ prognostic nutritional index, CONUT controlling nutritional status, CEA carcinoembryonic antigen, $C A$ carbohydrate antigen

immune-nutritional index, has previously been reported in many malignancies, including GC. In our study, we also observed that, CONUT score was able to detect more patients who would have a poor survival but not be identified by PNI. As shown in Table 2, we found that 137 patients $(22.2 \%)$ belonged to the low PNI group and the high CONUT group. Of note, in the low PNI group, CONUT score still effectively stratified CSS. Therefore, in the context of stage II-III GC, the CONUT score might exert more potent prognostic effect than did the PNI. This is partly attributed to the fact that there is greater emphasis placed on the total lymphocyte count in the CONUT score. Furthermore, total cholesterol concentration which is not evaluated in the PNI may play an important role as part of the CONUT score composite measure. Therefore, we speculated that the CONUT score might be a more comprehensive and superior predictor to identify nutritional risk than the PNI in GC. Maehara et al. enrolled 109 patients with lung cancer with obstructive pulmonary disease and found the CONUT score was an independent predictor of disease-free and overall survival [28]. Likewise, 


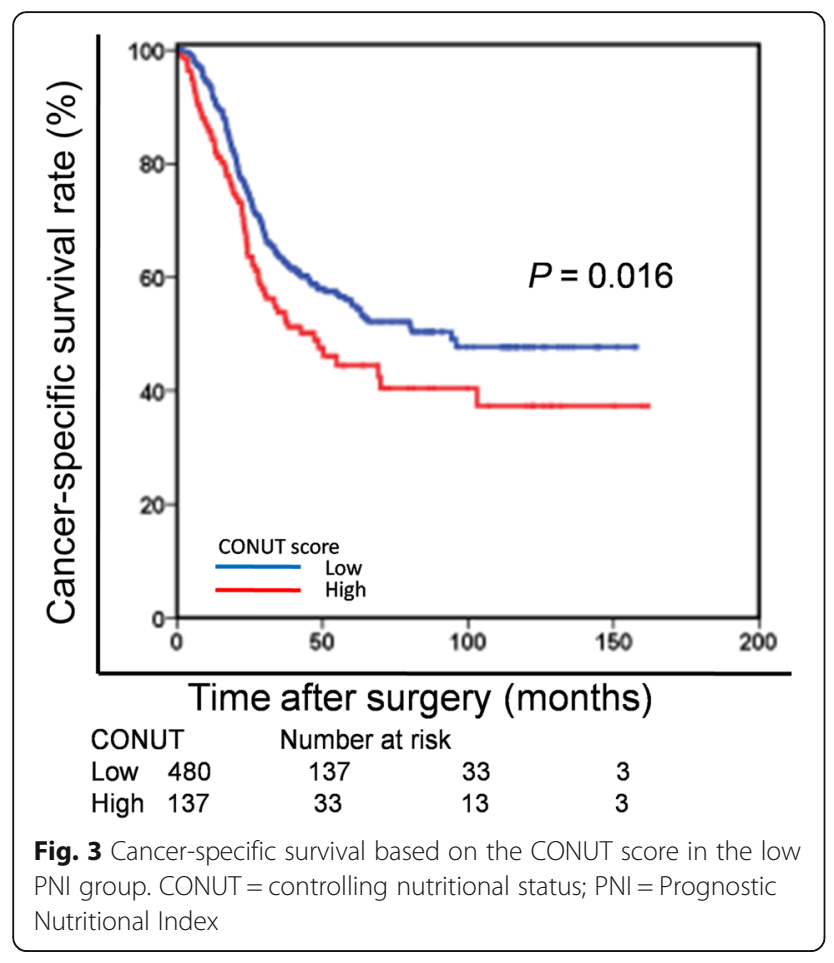

Maehara et al. evaluated and reported the prognostic value of CONUT score in 357 patients with hepatocellular carcinoma. They found that CONUT score was independently associated with overall survival, but not recurrence-free survival, in hepatocellular carcinoma patients undergoing curative resection [29].

Based on our study, it is thought that the preoperative CONUT score may be useful in the stratification of risk and tailoring individualize treatments. In clinical practice, patients with high CONUT score should receive more effective adjuvant therapy and shorten the follow-up interval. Furthermore, considering the promising results of targeted nutritional intervention, patients with high CONUT score may benefit from preoperative nutritional intervention [30-32]. However, up to now, the optimum nutritional intervention for improving the cancer-associated malnutrition has yet to be established. With all this in mind, we suggest that preoperative nutritional support based on the CONUT score should be evaluated in prospective randomized controlled studies.

Some limitations associated with our study warrant mention. First, it was a retrospective single-center rather than multicenter study. Thus, there might be potential selection bias for the inclusion of patients. Second, we did not have information on postoperative CONUT score and surgical complications. Future studies are needed to further explore. Third, different nutritional support after surgery was inevitable, and this might have confounded our results.

\section{Conclusions}

The CONUT score is independently associated with CSS in patients undergoing curative surgery followed by adjuvant chemotherapy for stage II-III GC. As a convenient, objective and noninvasive marker, it may be useful for treatment decision-making and improving follow-up performance.

\section{Additional files}

Additional file 1: Figure S1. Receiver operating characteristics curve for the CONUT score. CONUT = controlling nutritional status (TIF 2636 kb).

Additional file 2: Table S1. STROBE Statement-Checklist of items that should be included in the study (DOC $79 \mathrm{~kb}$ ).

\section{Abbreviations}

AJCC: American Joint Committee on Cancer; BMI: body mass index; CA: carbohydrate antigen; CEA: carcinoembryonic antigen; Cl: confidence interval; CONUT: controlling nutritional status; CSS: cancer-specific survival; $\mathrm{CT}$ : computed tomography; GC: gastric cancer; HR: hazard ratio; LVI: lymphatic vessel infiltration; PNI: prognostic nutritional index; ROC: receiver operating characteristic; STROBE: Strengthening the Reporting of Observational Studies in Epidemiology; TNM: tumor-nodes-metastasis

\section{Acknowledgments}

The authors are grateful to the people who kindly cooperated in our study.

\section{Availability of data and materials}

The datasets used and/or analysed during the current study are available from the corresponding author on reasonable request.

\section{Authors' contributions}

ZZW and SXW contributed to the conception and design of the study; ZDY, LEZ, CYB, LW, LXC and CYM performed the literature search, data extraction, quality assessment and statistical analyses; LXC composed the first draft of the manuscript; All authors read and critically revised the manuscript. All authors read and approved the final manuscript. All authors agreed to be accountable for all aspects of the work in ensuring that questions related to the accuracy or integrity of any part of the work are appropriately investigated and resolved.

\section{Ethics approval and consent to participate}

This study was performed in accordance with the principles embodied in the Declaration of Helsinki. The protocol and consent forms were reviewed and approved by the Ethical Committee of Sun Yat-sen University Cancer Center. Informed consent was deemed unnecessary by the Ethical Committee, and all information were anonymous.

\section{Consent for publication}

Not applicable.

\section{Competing interests}

The authors declare that they have no competing interests.

\section{Publisher's Note}

Springer Nature remains neutral with regard to jurisdictional claims in published maps and institutional affiliations.

\section{Author details}

${ }^{1}$ State Key Laboratory of Oncology in South China; Collaborative Innovation Center for Cancer Medicine, Sun Yat-sen University Cancer Center, Guangzhou 510060, China. ²Department of Gastric Surgery, Sun Yat-sen University Cancer Center, 651\# East Dongfeng road Guangzhou, 510060 Guangdong Province, People's Republic of China. ${ }^{3}$ Cancer Hospital of Shantou University Medical College, Shantou 515041, China. 
Received: 6 February 2018 Accepted: 20 June 2018

Published online: 28 June 2018

\section{References}

1. Chen W, Zheng R, Baade PD, Zhang S, Zeng H, Bray F, Jemal A, Yu XQ, He J. Cancer statistics in China, 2015. CA Cancer J Clin. 2016;66(2):115-32.

2. Ferlay J, Soerjomataram I, Dikshit R, Eser S, Mathers C, Rebelo M, Parkin DM, Forman D, Bray F. Cancer incidence and mortality worldwide: sources, methods and major patterns in GLOBOCAN 2012. Int J Cancer. 2015;136(5): E359-86.

3. Rahman R, Asombang AW, Ibdah JA. Characteristics of gastric cancer in Asia. World J Gastroenterol. 2014;20(16):4483-90.

4. Mori S, Usami N, Fukumoto K, Mizuno T, Kuroda H, Sakakura N, Yokoi K, Sakao Y. The significance of the prognostic nutritional index in patients with completely resected non-small cell lung Cancer. PLoS One. 2015;10(9): e0136897.

5. Sun KY, Xu JB, Chen SL, Yuan YJ, Wu H, Peng JJ, Chen CQ, Guo P, Hao YT, He YL. Novel immunological and nutritional-based prognostic index for gastric cancer. World J Gastroenterol. 2015;21(19):5961-71.

6. Liu X, Xu P, Qiu H, Xu D, Li W, Zhan Y, Li Y, Chen Y, Zhou Z, Sun X. Preoperative nutritional deficiency is a useful predictor of postoperative outcome in patients undergoing curative resection for gastric Cancer. Transl Oncol. 2016;9(6):482-8

7. Yim GW, Eoh KJ, Kim SW, Nam EJ, Kim YT. Malnutrition identified by the nutritional risk index and poor prognosis in advanced epithelial ovarian carcinoma. Nutr Cancer. 2016:68(5):772-9.

8. Secker DJ, Jeejeebhoy KN. Subjective global nutritional assessment for children. Am J Clin Nutr. 2007:85(4):1083-9.

9. Kang M, Chang $C T$, Sung HH, Jeon HG, Jeong BC, Seo SI, Jeon SS, Choi HY Lee HM. Prognostic significance of pre- to postoperative dynamics of the prognostic nutritional index for patients with renal cell carcinoma who underwent radical nephrectomy. Ann Surg Oncol. 2017;24(13):4067-75.

10. Ignacio de Ulibarri J, Gonzalez-Madrono A, de Villar NG, Gonzalez P, Gonzalez B, Mancha A, Rodriguez F, Fernandez G. CONUT: a tool for controlling nutritional status. First validation in a hospital population. Nutr Hosp. 2005;20(1):38-45.

11. Lien YC, Hsieh CC, Wu YC, Hsu HS, Hsu WH, Wang LS, Huang MH, Huang BS. Preoperative serum albumin level is a prognostic indicator for adenocarcinoma of the gastric cardia. J Gastrointest Surg. 2004;8(8):1041-8.

12. Miura K, Hamanaka K, Koizumi T, Kitaguchi Y, Terada Y, Nakamura D, Kumeda H, Agatsuma H, Hyogotani A, Kawakami S, et al. Clinical significance of preoperative serum albumin level for prognosis in surgically resected patients with non-small cell lung cancer: comparative study of normal lung, emphysema, and pulmonary fibrosis. Lung cancer (Amsterdam, Netherlands). 2017:111:88-95.

13. Lee $Y L$, Li WC, Tsai TH, Chiang HY, Ting CT. Body mass index and cholesterol level predict surgical outcome in patients with hepatocellular carcinoma in Taiwan - a cohort study. Oncotarget. 2016;7(16):22948-59.

14. Mantovani A, Allavena P, Sica A, Balkwill F. Cancer-related inflammation. Nature. 2008:454(7203):436-44.

15. Ishihara H, Kondo T, Yoshida K, Omae K, Takagi T, lizuka J, Tanabe K. Preoperative controlling nutritional status (CONUT) score as a novel predictive biomarker of survival in patients with localized urothelial carcinoma of the upper urinary tract treated with radical nephroureterectomy. Urol Oncol. 2017;35(9):539-e539.

16. Toyokawa T, Kubo N, Tamura T, Sakurai K, Amano R, Tanaka H, Muguruma K, Yashiro M, Hirakawa K, Ohira M. The pretreatment controlling nutritional status (CONUT) score is an independent prognostic factor in patients with resectable thoracic esophageal squamous cell carcinoma: results from a retrospective study. BMC Cancer. 2016;16:722.

17. Takagi K, Yagi T, Umeda Y, Shinoura S, Yoshida R, Nobuoka D, Kuise T, Araki $H$, Fujiwara T. Preoperative controlling nutritional status (CONUT) score for assessment of prognosis following hepatectomy for hepatocellular carcinoma. World J Surg. 2017;41(9):2353-60.

18. Kuroda D, Sawayama H, Kurashige J, Iwatsuki M, Eto T, Tokunaga R, Kitano Y, Yamamura K, Ouchi M, Nakamura K, et al. Controlling nutritional status (CONUT) score is a prognostic marker for gastric cancer patients after curative resection. Gastric Cancer. 2018;21(2):204-12.

19. Oh DY, Bang YJ. Adjuvant and neoadjuvant therapy for gastric cancer. Curr Treat Options in Oncol. 2013;14(3):311-20.
20. Fujitani K. Overview of adjuvant and neoadjuvant therapy for resectable gastric cancer in the east. Dig Surg. 2013;30(2):119-29.

21. Pinato DJ, North BV, Sharma R. A novel, externally validated inflammationbased prognostic algorithm in hepatocellular carcinoma: the prognostic nutritional index (PNI). Br J Cancer. 2012;106(8):1439-45.

22. Argiles JM. Cancer-associated malnutrition. Eur J Oncol Nurs. 2005; 9(Suppl 2):S39-50.

23. Van Cutsem E, Arends J. The causes and consequences of cancer-associated malnutrition. Eur J Oncol Nurs. 2005;9(Suppl 2):S51-63.

24. Mantzorou M, Koutelidakis A, Theocharis S, Giaginis C. Clinical value of nutritional status in Cancer: what is its impact and how it affects disease progression and prognosis? Nutr Cancer. 2017;69(8):1151-76.

25. Liang RF, Li JH, Li M, Yang Y, Liu YH. The prognostic role of controlling nutritional status scores in patients with solid tumors. Clinica chimica acta; international journal of clinical chemistry. 2017:474:155-8.

26. Yu M, Zheng HC, Xia P, Takahashi H, Masuda S, Takano Y, Xu HM. Comparison in pathological behaviours \& prognosis of gastric cancers from general hospitals between China \& Japan. Indian J Med Res. 2010;132:295-302.

27. Iseki Y, Shibutani M, Maeda K, Nagahara H, Ohtani H, Sugano K, Ikeya T, Muguruma K, Tanaka H, Toyokawa T, et al. Impact of the preoperative controlling nutritional status (CONUT) score on the survival after curative surgery for colorectal Cancer. PLoS One. 2015:10(7):e0132488.

28. Akamine T, Toyokawa G, Matsubara T, Kozuma Y, Haratake N, Takamori S, Katsura M, Takada K, Shoji F, Okamoto T, et al. Significance of the preoperative CONUT score in predicting postoperative disease-free and overall survival in patients with lung adenocarcinoma with obstructive lung disease. Anticancer Res. 2017;37(5):2735-42.

29. Harimoto N, Yoshizumi T, Sakata K, Nagatsu A, Motomura T, Itoh S, Harada $\mathrm{N}$, Ikegami T, Uchiyama $H$, Soejima $Y$, et al. Prognostic significance of preoperative controlling nutritional status (CONUT) score in patients undergoing hepatic resection for hepatocellular carcinoma. World J Surg. 2017:41(11):2805-12.

30. Nikniaz Z, Somi MH, Nagashi S, Nikniaz L. Impact of early enteral nutrition on nutritional and immunological outcomes of gastric Cancer patients undergoing gastrostomy: a systematic review and meta-analysis. Nutr Cancer. 2017;69(5):693-701.

31. $X u$ J, Zhong $Y$, Jing $D, W u Z$. Preoperative enteral immunonutrition improves postoperative outcome in patients with gastrointestinal cancer. World J Surg. 2006;30(7):1284-9.

32. Marin Caro MM, Laviano A, Pichard C. Nutritional intervention and quality of life in adult oncology patients. Clin Nutr (Edinburgh, Scotland). 2007;26(3): 289-301.

\section{Ready to submit your research? Choose BMC and benefit from:}

- fast, convenient online submission

- thorough peer review by experienced researchers in your field

- rapid publication on acceptance

- support for research data, including large and complex data types

- gold Open Access which fosters wider collaboration and increased citations

- maximum visibility for your research: over $100 \mathrm{M}$ website views per year

At BMC, research is always in progress.

Learn more biomedcentral.com/submissions 